\title{
Exposure to recipes in a food-based approach to increase egg and protein intake in community-dwelling older adults: a randomised controlled trial
}

\author{
E. van den Heuvel ${ }^{1}$, J.L. Murphy ${ }^{2}$ and K.M. Appleton ${ }^{1}$ \\ ${ }^{1}$ Research Centre for Behaviour Change, Department of Psychology and ${ }^{2}$ Faculty of Health and Social Sciences, \\ Bournemouth University, Poole, BH12 5BB
}

Dietary protein intake can have important impacts on health, physical abilities, muscle mass and strength ${ }^{(1)}$, and the prevalence of protein specific under-nutrition among older adults tends to be high ${ }^{(2)}$. When taking a food-based approach, increasing egg intake may facilitate protein intake in older adults. Compared to other protein rich foods, eggs are easy to cook, of long shelf life, soft texture and low cost ${ }^{(3,4)}$. Previous work ${ }^{(5,6)}$ indicates that older adults who eat more eggs think that eggs taste good and add variety to the diet. This suggests that adding flavour and more variety may encourage intakes in those who consume fewer eggs.

A randomized controlled intervention study was designed to increase egg and protein intake in community-dwelling adults aged 55 years and over. During the intervention, recipes for protein-rich egg-based meals and herbs/spice packets were provided to encourage the addition of flavour and variety to the diet. All participants received dietary information, and those randomized to the intervention group received 6 recipes and relevant herbs/spices every fortnight for 3 months. Dietary intake (Food Frequency Questionnaire ${ }^{(7)}$ ), body composition (Bioelectrical Impedance Analysis), handgrip strength, and physical functioning (Short Physical Performance Battery $(\mathrm{SPPB})^{(8)}$ ) were assessed at baseline, after the 3 month intervention and at 6 months (ClinicalTrials.gov registration: NCT02777918).

A total of 100 participants took part, including 54 females and 46 males, mean age $=70(\mathrm{SD}=7)$ years, range $55-97$ years. At baseline, egg intake was $22(\mathrm{SD}=16)$ eggs per month; daily protein intake was $92(\mathrm{SD}=32)$ grams; $9 \%$ could be classified as sarcopenic based on handgrip strength ${ }^{(9)}$; and $50 \%$ did not meet the protein recommendations for older adults ${ }^{(1)}$. After the 3 month intervention period, mean egg intake increased for both groups (intervention group: $23(\mathrm{SD}=17)$ to $28(\mathrm{SD}=20)$ eggs, control group: $21(\mathrm{SD}=15)$ to $25(\mathrm{SD}=20)$ eggs), and at 6 months, mean egg intake remained stable at $27(\mathrm{SD}=22)$ eggs for the intervention group, but returned to $22(\mathrm{SD}=15)$ eggs for the control group. Multiple linear regression controlling for several dietary and lifestyle characteristics showed no differences between groups at 3 months $($ Beta $=-.01, p=.84)$, and higher egg intake in the intervention than in the control group at 6 months $($ Beta $=-.12, p=.05)$. Dietary protein intake, physical functioning and strength were not significantly different between the groups at either time point. However, other significant predictors of higher egg intake at 6 months were a higher egg intake at baseline, a higher protein intake at 6 months and a higher age (smallest Beta $=.14, p=.04$ ).

This study shows that exposure to recipes, herb/spice packets, and dietary information can change eating behaviour in older adults up to 3 months after an intervention, while providing only dietary information failed to have this effect. An absence of impact on protein intakes may be explained by high baseline protein intakes, and the possibility that our egg-based meals replaced meals already high in protein. However, providing recipes as a strategy to encourage older adults to consume more eggs could be straightforward to implement in a larger population, and may be of benefit.

1. Bauer J, Biolo G, Cederholm T et al. (2013) J Am Med Dir Assoc 14, 542-559.

2. Hengeveld LM, Wijnhoven HA, Olthof MR et al. (2018) Am J Clin Nutr 107, 155-164.

3. Rousset S, and Jolivet P (2002) J Sens Stud 17, 61-75.

4. Lewis BR \& Bashin A (1988) Brit Food J 90, 172-177.

5. van den Heuvel E, Murphy JL, Appleton KM (2015) Proc Nutr Soc 74, 314.

6. van den Heuvel E, Murphy JL, Appleton KM (2016) Proc Nutr Soc 75, 196.

7. Clark H (2017) Scottish collaborative group food frequency questionnaire service. [accessed March 2017]. Available from: http://www.foodfrequency. org/

8. Guralnik JM, Simonsick EM, Ferrucci L et al. (1994) J Gerontol 49, M85-M94.

9. Cruz-Jentoft AJ, Baeyens JP, Bauer JM et al. (2010) Age Ageing 39, 412-423. 